\title{
Avaliação da aplicação dos métodos indiretos de predição do gradiente de pressão de poros em projetos de poços da Bacia Sergipe-Alagoas
}

\author{
An evaluation of the application of indirect methods of pore \\ pressure gradient prediction in well projects of the Sergipe-Alagoas Basin
}

\author{
Mariana do Nascimento Silva ${ }^{1}$, João Paulo Lobo dos Santos ${ }^{1}$, \\ Rafael de Castro Oliveira ${ }^{1}$, Itson Santos de Souza ${ }^{1}$, Acto de Lima Cunha ${ }^{1}$ \\ ${ }^{1}$ Núcleo de Engenharia de Petróleo, Universidade Federal de Sergipe - UFS, Avenida Marechal Rondon, s/n, \\ Jardim Rosa Elze, CEP 49100-000, São Cristóvão, SE, BR (maripetufs@gmail.com; jplobo@ufs.br; \\ rcastro.oliveira@petrobras.com.br; itson_ss@hotmail.com; acto@ufs.br)
}

Recebido em 19 de janeiro de 2017; aceito em 04 de maio de 2018

\begin{abstract}
Resumo
No cenário petrolífero atual, a exploração de petróleo em camadas com grandes profundidades é cada vez mais frequente. Com isso, um estudo mais completo das geopressões se faz necessário, visto que essas são as responsáveis pela manutenção da estabilidade do poço. Na previsão de geopressões são utilizados, geralmente, métodos indiretos, que consistem em correlações empíricas que foram calibradas para uma determinada área. Este trabalho teve como principal objetivo fazer uma análise comparativa e técnica das pressões de poros preditas na Bacia Sergipe-Alagoas, utilizando correlações empíricas calibradas para o Golfo do México. No cálculo dos gradientes de pressão de poros foram utilizados dados de dois poços, $\mathrm{X}$ e Y, fornecidos pela empresa $\mathrm{Z}$. Os resultados obtidos através dos métodos indiretos foram comparados com os dados de testes de formação realizados nos poços durante a perfuração. Os resultados obtidos mostraram que, para o poço $\mathrm{X}$, os valores preditos foram conclusivos e coerentes, pois os erros relativos médios dos gradientes de pressão de poros oscilaram entre 2,52 e 11,38\%, sendo esses valores próximos dos dados medidos nos testes de formação. Já para o poço Y, os resultados preditos foram representativos apenas no intervalo em que o gradiente de poros medido no teste de formação apresentou valor normal.
\end{abstract}

Palavras-chave: Geopressões; Pressões de poros; Métodos indiretos; Bacia Sergipe-Alagoas.

\begin{abstract}
In the current oil scenario, the exploration of layered oil at great depths is becoming increasingly frequent. Thus, a more complete study of geopressure is necessary, since it is responsible for maintaining the stability of wells. When predicting geopressures, indirect methods are usually used, which consist of empirical correlations that have been calibrated for a certain area. This work's main objective was to make a comparative and technical analysis of the pore pressures predicted in the Sergipe-Alagoas Basin, using calibrated empirical correlations for the Gulf of Mexico. In the calculation of the pore pressure gradients, data from two wells, $\mathrm{X}$ and $\mathrm{Y}$, provided by company $\mathrm{Z}$ were used. The results obtained using the indirect methods were compared with the data from formation tests that were performed in the wells during drilling. The results showed that, for well $\mathrm{X}$, the predicted values were conclusive and coherent, as the mean relative errors of the pore pressure gradients ranged from $2.52 \%$ to $11.38 \%$, and these values are close to the data measured in the training. As for well Y, the predicted results were representative only in the interval in which the pore gradient measured in the formation test had a normal value.
\end{abstract}

Keywords: Geopressure; Pore pressure; Indirect methods; Sergipe-Alagoas Basin. 


\section{INTRODUÇÃO}

O conhecimento das geopressões é de fundamental importância para o planejamento da construção e a estabilidade do poço. O termo "geopressões" pode ser usado para designar a pressão de formação ou de poros e pode, também, compreender a geomecânica. No primeiro caso são analisadas as pressões de fluidos contidos nos poros das rochas localizadas no subsolo; e o segundo caso diz respeito ao comportamento mecânico de todos os materiais geológicos e suas reações dos campos de força manifestados no meio físico. Simplificadamente, verifica-se que o objetivo do estudo das geopressões é a determinação das pressões de poros, de fratura e de colapso que formam a janela operacional de um poço (Rocha e De Azevedo, 2009).

Os dados completos e confiáveis das geopressões são responsáveis pelo sucesso na execução da perfuração de um poço. Caso não se tenha uma análise confiável do campo de geopressões antes da perfuração, na condição estática, poderão ser necessárias paradas durante a perfuração, que podem gerar altos custos. Essas paradas são causadas, na maioria das vezes, por aprisionamento de coluna, torques elevados — que podem atingir os limites mecânicos do equipamento - , colapso total do poço e influxo de fluido da formação para dentro do poço, podendo ser controlado (kick) ou descontrolado (blowout), os quais podem ocasionar perdas humanas, prejuízos econômicos e danos ambientais (Pereira, 2007; Rocha e De Azevedo, 2009). Entretanto, esses problemas podem ser minimizados quando se dispõe de ferramentas que permitem determinar os gradientes de geopressões de forma mais confiável.

Um fator relevante é que, na locação de um poço pioneiro, os únicos dados disponíveis são provenientes do perfil sísmico. Dessa forma, as curvas de geopressões são obtidas a partir de correlações feitas com a velocidade sísmica. $\mathrm{Na}$ análise da curva de sobrecarga, vários métodos relacionam diretamente a densidade das formações com a velocidade sísmica, observando que para cada área existe um parâmetro de calibração ajustado. Já no estudo da pressão de poros, as correlações baseiam-se, unicamente, no mecanismo de subcompactação, que consiste no aumento do gradiente de pressão de poros com o aumento da pressão de sobrecarga.

Alguns indicadores qualitativos da perfuração podem ser utilizados de forma quantitativa na avaliação da pressão de poros, conforme será visto mais adiante. Os mais comuns são os perfis elétricos, como densidade e sônico; a velocidade sísmica; e parâmetros baseados na normalização da taxa de penetração (expoente d).

$\mathrm{Na}$ literatura existem vários métodos para a estimativa da pressão de poros correlacionando esses indicadores, conforme Rocha e De Azevedo (2009). A partir desses indicadores, diversas correlações foram elaboradas.
Hottman e Johnson (1965) desenvolveram um modelo empírico a partir da geração de um gráfico que correlaciona os desvios do trend (linha com tendência de compactação normal) com medições in situ de pressão de poros. Pennebaker (1968) desenvolveu um método empírico, afirmando que as velocidades das ondas sísmicas em um meio são influenciadas pela litologia, idade geológica e pressão de poros. Eaton (1975) desenvolveu um método similar ao da profundidade equivalente (PE), com a diferença de utilizar os desvios do trend para estimar as tensões efetivas. Holbrook et al. (1995) descreveram como estimar a pressão de poros em qualquer bacia sedimentar independentemente do tipo de litologia existente. Bowers (1995) gerou dois modelos para fazer a previsão, levando em conta a ação de dois mecanismos, subcompactação e subcompactação com expansão de fluidos. Sayers et al. (2002) utilizaram as velocidades intervalares estimadas, assim como os modelos de Bowers (1995) e Eaton (1975), para fazer a estimativa da pressão de poros. Dutta (2002) mostrou como são estimadas as pressões anormais de forma mais clara e objetiva, utilizando modelos mecânico-físicos. Sayers et al. (2002) concluíram ainda que a equação de Issler (1992) é a mais utilizada para estimar a porosidade em folhelhos a partir de registros sônicos ou tempos intervalares. Doyen e Gaudoin (2004) apresentaram um modelo mecânico-físico, baseado no modelo de Bowers (1995), levando em conta a porosidade e o conteúdo de argilas da formação. Santos et al. (2015) avaliaram a aplicabilidade das correlações empíricas desenvolvidas para o Golfo do México no estudo das geopressões na Bacia Sergipe-Alagoas e concluíram que o expoente de Eaton tem grande influência no cálculo mais confiável do gradiente de poros.

O principal objetivo deste trabalho foi avaliar as metodologias indiretas para a predição do gradiente de poros, bem como sua aplicabilidade em projetos de poços na Bacia Sergipe-Alagoas. Para isso, na validação ou não dos métodos indiretos, foram comparados os resultados obtidos das equações empíricas com os dados diretos de testes realizados em poços da área em estudo.

\section{GRADIENTE DE PRESSÃO DE POROS}

A pressão de poros pode ser definida como a pressão exercida pelos fluidos que estão dentro dos espaços vazios (poros) das rochas. A quantificação da pressão de poros é extremamente necessária para a construção da janela operacional, pois delimita as tensões aplicadas na formação. É a partir do conhecimento da pressão de poros que se escolhe o peso do fluido mais adequado para uma manutenção segura do campo de pressões de um poço (Santos et al., 2015).

Quanto à intensidade, o gradiente de pressão de poros (Gp) pode ser classificado em anormalmente baixo, normal, 
anormalmente alto e alta sobrepressão, conforme é apresentado na Tabela 1.

Devido à complexidade em relação ao grande número de variáveis envolvidas, a obtenção do Gp nem sempre é fácil e precisa. Os processos para obtenção do Gp são divididos em medições diretas, feitas em zonas permeáveis, e medições indiretas, feitas em zonas impermeáveis, preferencialmente nos folhelhos (Pereira 2007).

\section{Medição direta}

Como já mencionado, as medições diretas são feitas em intervalos permeáveis, ou seja, em zonas produtoras ou potencialmente produtoras. Por meio das medições diretas são feitas as avaliações do reservatório e dos fluidos contidos no poço. Além disso, servem como parâmetro de validação e de calibração para os métodos indiretos. O único contratempo desse tipo de medição é que os registros diretos são pontuais, ou seja, não conseguem predeterminar o comportamento da pressão ao longo de todas as camadas atravessadas pelo poço (Rocha e De Azevedo, 2009).

Os principais testes para as medições de pressão de poros funcionam de maneira similar, sendo as únicas diferenças a forma de coleta das amostras e o tempo de duração de cada teste. Os testes mais utilizados são: teste de formação, repeated formation test (RFT) e formation pressure while drilling (FPWD). O RFT é um teste muito utilizado por ser mais simples e rápido, quando comparado ao teste de formação, uma vez que é feito a cabo ao invés de coluna de perfuração, acelerando o processo. A ferramenta de teste é composta por um sistema de válvulas e câmaras, possibilitando a medição da pressão estática. Já o FPWD é realizado em tempo real durante a perfuração do poço, utilizando um conjunto de sensores acima da broca, no entanto, é um método mais caro que o RFT (Santos et al., 2015).

\section{Medição indireta}

Os métodos indiretos para predição do Gp foram desenvolvidos para serem utilizados em formações impermeáveis, como os folhelhos, que por serem argilosas e com baixa permeabilidade, são bastante influenciadas pelo efeito de subcompactação. Os mecanismos para obtenção dos

Tabela 1. Classificação dos gradientes de pressão de poros.

\begin{tabular}{ll}
\hline Anormalmente baixo & $\mathrm{Gp}<8,5 \mathrm{lb} / \mathrm{gal}$ \\
Normal & $8,5 \mathrm{lb} / \mathrm{gal}<\mathrm{Gp}<9 \mathrm{lb} /$ gal \\
& $9,1 \mathrm{lb} / \mathrm{gal}<\mathrm{Gp}<90 \%$ gradiente \\
Anormalmente alto & de sobrecarga \\
Alta sobrepressão & $\mathrm{Gp}>90 \%$ gradiente de sobrecarga \\
\hline
\end{tabular}

Gp: gradiente de poros. registros indiretos baseiam-se em perfis elétricos, dados de velocidades sísmicas ou outros parâmetros indicadores da porosidade. O primeiro passo é a determinação de um trecho de tendência cujo efeito da compactação seja normal, em outras palavras, o trecho onde ocorreu a diminuição da porosidade com o aumento da profundidade (Rocha e De Azevedo, 2009). A Figura 1 mostra como esses indicadores podem ser utilizados, de forma quantitativa, na identificação da passagem de uma zona de compactação normal para outra zona, anormalmente pressurizada. A mudança de uma zona para outra é caracterizada pelo desvio do trend com o aumento da profundidade.

Com o objetivo de simplificar a função de compactação normal, foi feita uma aproximação para uma reta num gráfico semilogarítimico, conforme mostrado pelas Equações 1 e 2 (Ferreira 2010).

$m=\frac{\log \left(\frac{v a l_{2}}{v a l_{1}}\right)}{D_{2}-D_{1}}$

$v a l_{\mathrm{n}}=v a l_{1} 10^{m\left(D-D_{1}\right)}$

Em que:

$m=\mathrm{o}$ coeficiente angular da reta de tendência normal em um gráfico semilog;

$v a l_{1}$ e $v a l_{2}=$ valores observados do parâmetro indicador de porosidade, onde a compactação foi normal;

$D_{1}$ e $D_{2}=$ profundidades equivalentes aos pontos $\mathrm{val}_{1} \mathrm{e}$ $\operatorname{val}_{2}(\mathrm{~m})$

$D=$ profundidade de interesse desejada $(\mathrm{m})$;

$v a l_{\mathrm{n}}=\mathrm{o}$ valor da reta de tendência normal na profundidade de interesse $D$.

Uma maneira simplificada de se traçar uma curva de compactação normal não linear é utilizando a metodologia chamada trends curvos. Essa metodologia assume

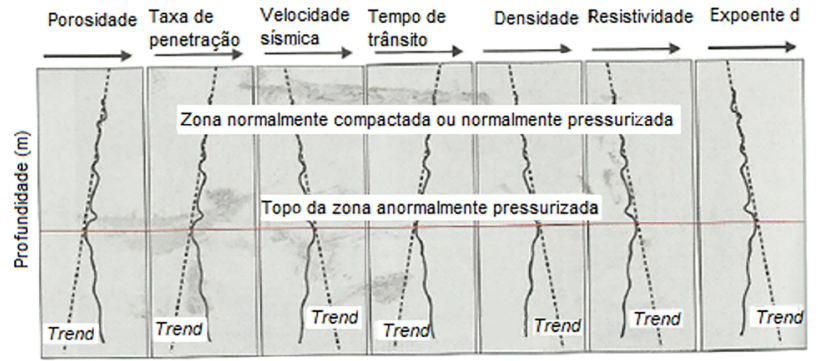

Fonte: Modificado de Rocha e De Azevedo (2009).

Figura 1. Comportamento dos indicadores quantitativos de mudança de zona normalmente compactada para anormalmente pressurizada. 
que a tendência de compactação é não linear com a profundidade e, nesse caso, são escolhidos dois pontos na curva do perfil sônico, nos quais se deseja que a curva de compactação normal passe, conforme pode ser visto nas Equações 3, 4 e 5.

$\Delta t_{n}=a \log (D)+b$

$a=\frac{\left(\Delta t_{2}-\Delta t_{1}\right)}{\log \left(\frac{D_{2}}{D_{1}}\right)}$

$b=\Delta t_{1}-a \log \left(D_{1}\right)$

Em que:

$\Delta t_{1}$ e $\Delta t_{2}=$ tempos de trânsito escolhidos em um gráfico semi$\log$ correspondente à zona de compactação normal $(\mu \mathrm{s} / \mathrm{ft})$; $D_{1}$ e $D_{2}=$ profundidades equivalentes aos tempos $\Delta t_{1} \mathrm{e} \Delta t_{2}(\mathrm{~m})$; $a=$ a constante de declínio do trend de compactação normal; $b=\mathrm{o}$ valor do trend de compactação normal no fundo do mar.

Após o traçado da curva de compactação normal, o passo seguinte é determinar o Gp. Apesar da existência de vários métodos, os principais a serem abordados neste trabalho são: da razão (ratio), da PE e o mais comum no setor petrolífero, o método de Eaton.

\section{Método da razão}

O método da razão (ratio) baseia-se na hipótese de que a pressão de poros, numa certa profundidade, é proporcional ao gradiente normal da área, sem levar em conta o efeito da pressão de sobrecarga, tornando-o um método mais simplificado. A Equação 6 demonstra esse comportamento (Rocha e De Azevedo, 2009).

$G_{p}=G_{N}\left(\frac{v a l_{o}}{v a l_{n}}\right)^{m}$

Em que:

$G_{\mathrm{P}}=\mathrm{o}$ gradiente de pressão de poros (lb/gal);

$G_{\mathrm{N}}=\mathrm{o}$ valor do gradiente de pressão de poros normal da área (lb/gal);

$v a l_{\mathrm{n}}=\mathrm{o}$ valor da reta normal;

$v a l_{\mathrm{o}}=\mathrm{o}$ valor observado do parâmetro;

$m=\mathrm{o}$ expoente da área.

\section{Método da profundidade equivalente}

$\mathrm{O}$ método da profundidade equivalente (PE) foi proposto por Foster (1966). O valor da porosidade anormal gerada pela subcompactação é refletido em condições normais de compactação, numa profundidade denominada de "profundidade equivalente". Esse valor permite estimar a tensão efetiva, a qual é a mesma nas duas regiões (normal e anormalmente compactada). A partir das Equações 7 e 8, determina-se a pressão de poros através do método em questão.

$P_{P A}=P_{P B}+\left(\sigma_{O V A}-\sigma_{O V B}\right)$

$G_{p A}=G_{O V A}-\frac{D_{B}}{D_{A}}\left(G_{O V B}-G_{p B}\right)$

Em que:

$P_{\mathrm{PA}}=$ a pressão de poros na profundidade A (psi);

$P_{\mathrm{PB}}=$ a pressão de poros na profundidade B (psi);

$\sigma_{\mathrm{A}}=$ a tensão de sobrecarga em A (psi);

$\sigma_{\mathrm{B}}=$ a tensão de sobrecarga em B (psi);

$G_{\mathrm{ovA}}=\mathrm{o}$ gradiente de sobrecarga na profundidade A (lb/gal);

$G_{\mathrm{ovB}}=\mathrm{o}$ gradiente de sobrecarga na profundidade B (lb/gal);

$D_{\mathrm{A}}=$ a profundidade do ponto A (m);

$D_{\mathrm{B}}=$ a profundidade do ponto $\mathrm{B}(\mathrm{m})$.

\section{Método de Eaton}

O cálculo do Gp pelo método de Eaton depende da pressão de sobrecarga, da pressão de poros normal, da razão entre o valor do parâmetro observado e o valor na linha de tendência de compactação normal e do expoente escolhido. Para o Golfo do México, local para o qual a correlação foi elaborada, o valor do expoente é 3 . Esse é considerado o método mais utilizado na indústria do petróleo para predição indireta do gradiente de poros e é regido pela Equação 9 (Eaton, 1975).

$G_{p}=G_{O V}-\left[\left(G_{O V}-G_{N}\right)\left(\frac{\Delta t_{n}}{\Delta t_{o}}\right)^{3}\right]$

Em que:

$G_{\mathrm{ov}}=$ o gradiente de sobrecarga (lb/gal);

$\Delta t_{\mathrm{o}}=\mathrm{o}$ tempo de trânsito observado $(\mu \mathrm{s} / \mathrm{ft})$;

$\Delta t_{\mathrm{n}}^{\mathrm{o}}=\mathrm{o}$ valor da reta normal para o tempo de trânsito $(\mu \mathrm{s} / \mathrm{ft})$.

\section{METODOLOGIA}

No presente trabalho, foram utilizados dados de dois poços localizados na Bacia Sergipe-Alagoas. Obedecendo ao critério de confidencialidade na utilização dos dados, os poços foram chamados de poço $\mathrm{X}$ e poço $\mathrm{Y}$, sendo os mesmos fornecidos pela empresa $Z$. Os dois poços estão locados 
na mesma formação produtora. Entretanto, a profundidade do intervalo de interesse difere um do outro de forma que, em termos de profundidade, o poço $\mathrm{Y}$ pode ser considerado como uma continuidade vertical do poço X. Os dados dos poços utilizados estão disponíveis em De Andrade (2014).

Os dados disponíveis são da perfilagem e dos testes de formação (RFT), obtidos através da descida de ferramentas a cabo nos poços $\mathrm{X}$ e Y. Eles foram manipulados no Microsoft Office Excel 2007 e, em seguida, selecionados os perfis de interesse para este trabalho, a saber, os perfis sônicos e densidade (RHOB). Para a densidade das formações, foi utilizado o valor médio a cada $10 \mathrm{~m}$ de profundidade, visto que as informações disponíveis da perfilagem eram dadas a cada $10 \mathrm{~cm}$ de profundidade, gerando um volume muito grande de dados.

Inicialmente, para o cálculo dos gradientes de sobrecarga e de poros, foram utilizados os parâmetros calibrados para o Golfo do México, visto que as correlações empíricas disponíveis foram elaboradas para aquela área. Diante disso, é possível fazer uma comparação entre os gradientes de poros calculados, através dos três métodos existentes, e os gradientes medidos durante a perfuração.

Para a predição do gradiente de poros, primeiramente, se faz necessário o cálculo do gradiente de sobrecarga, que é feito com auxílio dos perfis que fornecem dados da densidade da formação. Esses dados foram obtidos através do perfil RHOB, que fornece os valores diretos das densidades sem necessidade de utilizar equações empíricas. Posteriormente, para o cálculo do gradiente de poros, foram utilizadas as Equações 6, 8 ou 9. Vale ressaltar que, como os perfis não foram corridos nas regiões superficiais, a densidade das formações superficiais foi considerada como $1,7 \mathrm{~g} / \mathrm{cm}^{3}$ (valor médio), baseado na literatura, que apresenta valores de densidade variando entre 1,5 e $1,9 \mathrm{~g} / \mathrm{cm}^{3}$, conforme proposto por Rocha e De Azevedo (2009).

O primeiro passo para o cálculo do gradiente de poros é a elaboração da curva de tendência de compactação normal. Para isso, utilizaram-se os dados disponíveis e o Microsoft Office Excel 2007 para a construção de um gráfico do perfil sônico no intervalo onde os dados foram coletados. Para traçar a curva de compactação normal, utilizou-se o método dos trends, linear e curvos. A Figura 2 mostra o fluxograma utilizado para predição do gradiente de poros pelos três métodos avaliados.

No cálculo do gradiente de poros foram utilizados os métodos 1, 2 e 3, sendo: o método da razão (ratio), o método da PE e o método de Eaton, respectivamente. O valor do gradiente de poros normal da área em estudo utilizado foi de $8,75 \mathrm{lb} /$ gal, que corresponde à média do intervalo que a literatura considera como normal.

Os dados de testes de formação obtidos para os poços $\mathrm{X}$ e Y estão apresentados nas Tabelas 2 e 3, respectivamente.

$\mathrm{Na}$ análise de erros, o cálculo dos erros relativo e absoluto tem como objetivo garantir confiabilidade aos modelos aplicados na obtenção dos gradientes. Dessa forma, a verificação do erro é uma etapa de extrema importância no estudo de dados obtidos através de métodos indiretos.

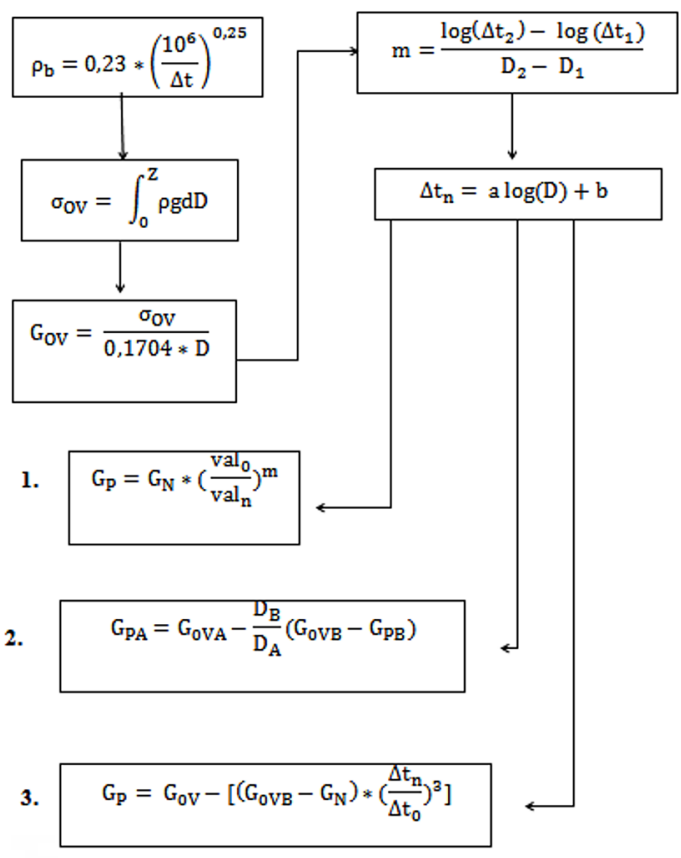

Fonte: Adaptado de Santos et al. (2015).

Figura 2. Fluxograma de cálculo da janela operacional.

Tabela 2. Dados de repeat formation test (poço X).

\begin{tabular}{cc}
\hline Profundidade $(\mathrm{m})$ & Gradiente de poros (medido) \\
\hline 953,2 & 8,35 \\
993,2 & 8,34 \\
1023,9 & 8,35 \\
\hline
\end{tabular}

Tabela 3. Dados de repeat formation test (poço Y).

\begin{tabular}{cc}
\hline Profundidade $(\mathrm{m})$ & Gradiente de poros (medido) \\
\hline $1.831,1$ & 8,18 \\
$1.844,7$ & 8,45 \\
$1.847,4$ & 8,34 \\
$1.855,3$ & 7,86 \\
$1.857,9$ & 7,69 \\
$1.871,9$ & 5,56 \\
$1.882,4$ & 6,53 \\
$1.923,2$ & 7,00 \\
$1.925,8$ & 6,99 \\
$1.939,6$ & 6,71 \\
$1.943,6$ & 6,68 \\
$1.974,7$ & 5,41 \\
$1.975,3$ & 5,45 \\
$2.012,4$ & 8,03 \\
$2.020,1$ & 6,46 \\
$2.121,9$ & 6,14 \\
\hline
\end{tabular}


O erro absoluto (EA) é considerado um erro médio para cada poço, definido pela Equação 10.

$E_{A}=\sum_{i=1}^{N}\left(\frac{P P D_{i}-P_{P_{i}}}{N}\right)$

Em que:

$P P D_{\mathrm{i}}=$ a pressão de poros determinada nos testes de RFT na profundidade $i(\mathrm{lb} / \mathrm{gal})$;

$P_{\mathrm{Pi}}=$ a pressão de poros estimada na profundidade $i(\mathrm{lb} / \mathrm{gal})$;

$N=$ o número de incremento de profundidades.

O erro relativo (ER) para cada poço se dá conforme a Equação 11.

$E R(\%)=100 \frac{\sum_{i=1}^{N}\left(\frac{P P D_{i}-P_{P_{i}}}{P P D_{i}}\right)}{N}$

\section{RESULTADOS E DISCUSSÃO}

A apresentação dos resultados obtidos foi realizada, separadamente, para cada poço, com base nos métodos indiretos de cálculo de gradiente de poros utilizados e, inicialmente, foi analisado o poço X. A partir dos dados diretos do perfil RHOB, obteve-se os valores do gradiente de sobrecarga.

Para o cálculo do gradiente de poros, foi construída a curva de compactação normal não linear, utilizando a metodologia dos trends linear e curvos. Os pontos utilizados devem ser escolhidos de modo a fazer com que o trend passe sobre a região assumida como normalmente compactada e, de acordo com Rocha e De Azevedo (2009), devem ser escolhidos pontos nas profundidades mais rasas, visto que são camadas normalmente compactadas. A finalidade da curva de compactação normal é fornecer o comportamento do logaritmo do tempo de trânsito em relação à profundidade de interesse. Para uma melhor visualização da tendência de compactação, o eixo x já vem com valores diretos do log $(\Delta \mathrm{T})$ versus profundidade, conforme mostrado na Figura 3.

Para o poço $\mathrm{X}$, os pontos escolhidos são referentes às profundidades 347 e $607 \mathrm{~m}$, conforme indicados na Figura 3, os quais obedecem à tendência da curva de compactação normal. O critério para escolha dos pontos leva em consideração a parte mais compactada da curva, que é o trecho em que o tempo de trânsito diminui à medida que a profundidade aumenta, ou seja, está ocorrendo uma redução da porosidade com o aumento da profundidade. Para uma melhor visualização, foi mostrada a tendência de compactação até a profundidade de $650 \mathrm{~m}$ (Figura 3).

Posteriormente à escolha dos dois pontos, calculou-se o coeficiente angular da reta - dado imprescindível no cálculo dos parâmetros -, $\Delta t_{\mathrm{n}}$ (linear) e $\Delta t_{\mathrm{n}}$ (trend curvo), responsáveis pela obtenção do Gp pelos métodos de Eaton e razão. O coeficiente angular da reta para o trend linear foi de $1,76 \times 10^{-4}$, enquanto que, para o trend curvo, os coeficientes angulares e lineares foram de -46 e 227,9; respectivamente.

A partir dos gradientes de poros calculados pelos três métodos utilizados neste trabalho, foi possível construir a curva de Gp versus profundidade, conforme mostrado na Figura 4. Especialmente, para o método de Eaton, as comparações foram feitas levando em consideração as metodologias lineares e trend curvo, ambas utilizando o expoente igual a 3 , que é o valor para o local onde as correlações foram elaboradas. O comparativo dos dados preditos por cada método indireto foi feito, primeiramente, de forma qualitativa, ou

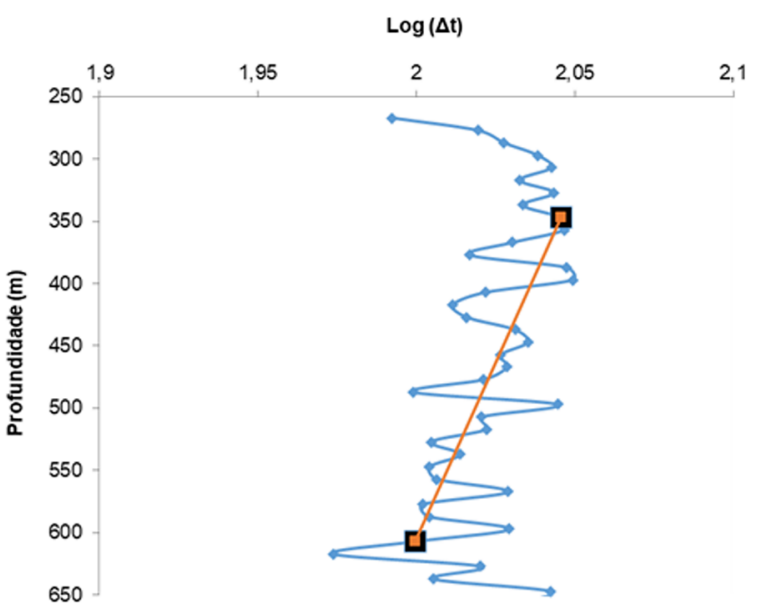

Figura 3. Curva de tendência de compactação normal do poço $X$.

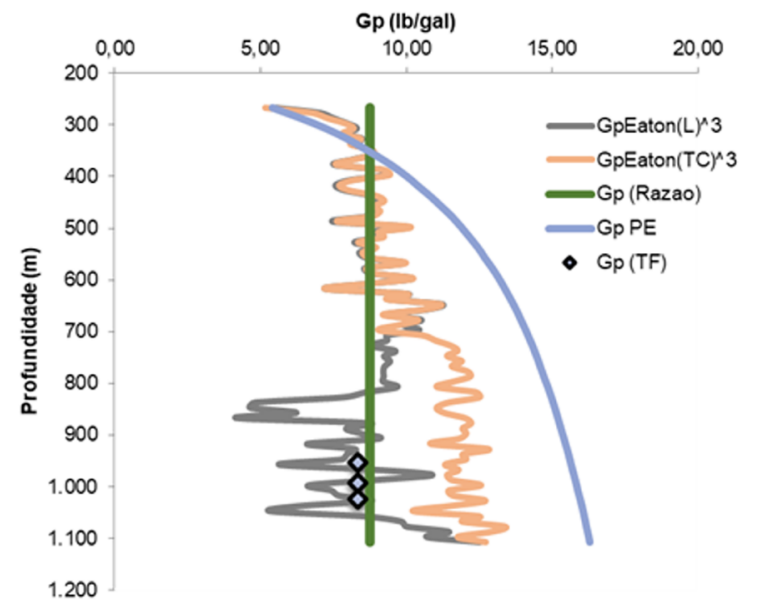

GP: gradiente de poros; L: linear; TC: trend curvo; PE: profundidade equivalente; TF: teste de formação.

Figura 4. Janela operacional dos métodos indiretos do poço $\mathrm{X}$ utilizando o expoente 3 para o método de Eaton. 
seja, observando qual(is) das curvas se aproxima(m) mais dos valores dos testes de formação.

$\mathrm{Na}$ Figura 4, é possível constatar que o método de Eaton com trend linear e expoente 3 e o método da razão foram os que mais se aproximaram dos valores medidos no teste de RFT. Dessa forma, em tese, os dois métodos são os mais apropriados para o cálculo indireto do Gp na Bacia Sergipe-Alagoas. No entanto, vale ressaltar que os resultados obtidos para o método da razão não são coerentes, visto que apresenta um valor de gradiente de poros constante para todas as profundidades, indo de encontro com o mecanismo da subcompactação. Se levarmos em consideração que o Gp é função do peso das camadas de rochas sobrepostas e que aumenta com a profundidade, o normal seria que o mesmo apresentasse variação com o aumento da profundidade, o que não ocorreu.

Por outro lado, comparando as metodologias de trend curvo e linear, os dois métodos apresentam resultados similares para profundidades menores, visto que, até cerca de 700 metros, as duas curvas se sobrepõem, ou seja, dentro da zona de compactação normal os dois métodos conseguiram prever bem o gradiente de poros. No entanto, para profundidades maiores, onde se encontra a zona de interesse e a compactação anormal, o trend linear é o que mais se aproxima dos valores registrados nos testes, sendo os mais precisos na previsão.

O mesmo procedimento foi repetido para a determinação do gradiente de poros pelo método de Eaton. No entanto, ocorreu uma variação no seu expoente (valor utilizado igual a 1), visto que, de acordo com Santos et al. (2015), a diferença entre os valores de gradiente de poros preditos e medidos diminui quando esse expoente é próximo de 1 para as bacias brasileiras. A Figura 5 mostra a comparação entre os métodos.

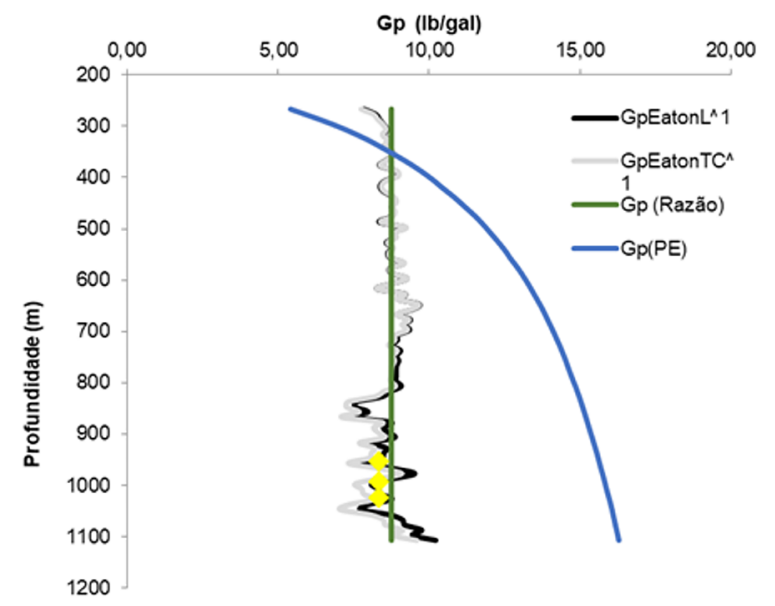

GP: gradiente de poros; L: linear; TC: trend curvo; PE: profundidade equivalente.

Figura 5. Janela operacional dos métodos indiretos do poço $\mathrm{X}$ utilizando o expoente 1 para o método de Eaton.
Na Figura 5, considerando que o parâmetro de Eaton é igual a 1, ambas metodologias com trend linear e curvo geraram resultados muito próximos aos valores medidos nos testes de formação, não havendo diferenças significativas, o que acarretou erros pequenos e próximos entre eles. Isso mostra que a metodologia dos trends curvo é mais sensível à mudança no expoente, quando comparada com o trend linear. Como pode ser visto nas Figuras 4 e 5, os dados preditos pelo método da profundidade equivalente não são apropriados para efeitos de comparação, pois estão completamente discrepantes dos valores medidos nos testes de RFT, assim como dos valores preditos pelos demais métodos indiretos. Nesse caso, não se recomenda a utilização desse método na previsão de gradiente de poros na Bacia Sergipe-Alagoas.

Para que seja possível fazer uma análise quantitativa, foram calculados os erros absolutos e relativos para cada método. Quantitativamente, os erros gerados entre os dados calculados em todos os métodos e os dados medidos no teste de formação são apresentados na Tabela 4.

Da Tabela 4, é possível verificar que os métodos mais apropriados para previsão do gradiente de poros, quando levados em consideração os erros, são o método de Eaton expoente 1 (linear e trend curvo) e o método da razão. No entanto, vale ressaltar que, apesar do ER médio para o método da razão ter sido baixo $(4,83 \%)$, ele não é adequado para ser aplicado na Bacia Sergipe-Alagoas pelos motivos já discutidos na análise qualitativa.

Dessa forma, o único método que apresentou resultados coerentes e que pode ser indicado para a predição do gradiente de poros no local em estudo é o método de Eaton. Os resultados mostram que é necessária uma análise qualitativa e quantitativa para a escolha do melhor método a ser utilizado na predição do gradiente de poros. Além disso, os resultados obtidos com a mudança do expoente estão de acordo com Santos et al. (2015).

Todas as análises efetuadas para o poço $\mathrm{X}$ foram repetidas para o poço $\mathrm{Y}$, nas quais o primeiro passo foi a determinação da curva de compactação normal, conforme apresentado na Figura 6. Para o cálculo do Gp do poço Y, foram utilizados os mesmos parâmetros e correlações do poço X,

Tabela 4. Análise de erro total do poço $X$.

\begin{tabular}{lcc}
\hline Método indireto & $\begin{array}{c}\text { Erro absoluto } \\
\text { (médio) }\end{array}$ & $\begin{array}{c}\text { Erro relativo \% } \\
\text { (médio) }\end{array}$ \\
\hline EatonTC (expoente 3) & 3,70 & 44,32 \\
EatonTC (expoente 1) & 0,52 & 6,23 \\
EatonL (expoente 3) & 0,95 & 11,38 \\
EatonL (expoente 1) & 0,21 & 2,52 \\
Razão & 0,40 & 4,83 \\
PE & 7,45 & 89,30 \\
\hline
\end{tabular}

TC: trend curvo; L: linear; PE: profundidade equivalente. 
sendo a curva de compactação normal (Figura 6) a única diferença entre eles. A curva de compactação foi construída levando em consideração os dados de perfilagem que variam de um poço para o outro. O coeficiente angular da reta para o trend linear foi de $9,79 \times 10^{-5}$, enquanto que para o trend curvo, os coeficientes angulares e lineares foram de $-66,3$ e 292,3; respectivamente.

Analisando o comportamento dos resultados das curvas de gradientes de poros versus profundidade, conforme mostrado nas Figuras 7 e 8, observou-se um arranjo semelhante

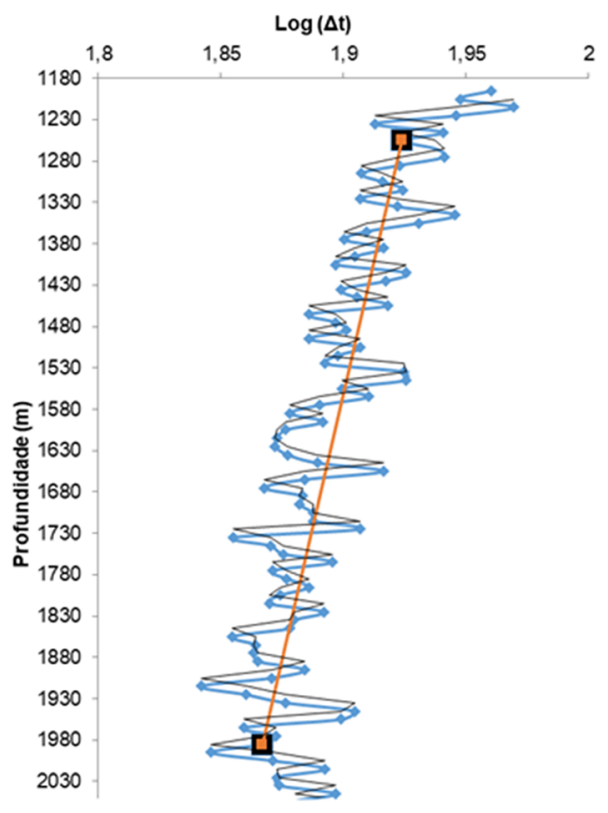

Figura 6. Curva de tendência de compactação normal do poço $Y$.

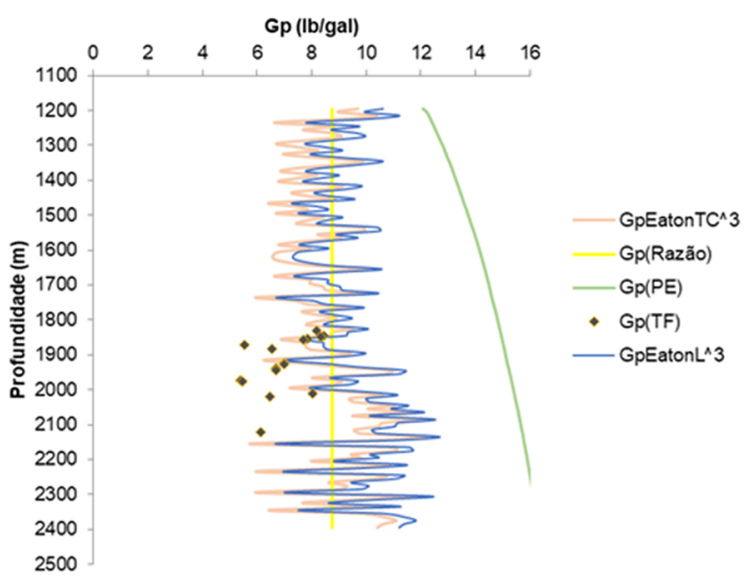

GP: gradiente de poros; TC: trend curvo; PE: profundidade equivalente; TF: teste de formação; L: linear.

Figura 7. Janela operacional dos métodos indiretos do poço $\mathrm{Y}$ utilizando o expoente 3 para o método de Eaton. ao poço anterior para todo o intervalo e não apenas para profundidades rasas, já que para os dois expoentes (1 ou 3) e nas duas metodologias (linear ou trend curvo), suas curvas foram muito próximas para todas as profundidades. Esse comportamento diferente na sobreposição das duas curvas, quando comparado com o poço $\mathrm{X}$, pode estar relacionado à profundidade da camada que, nesse caso, é maior e poderia já estar sob um regime de compactação anormal. Como já discutido, de acordo com Rocha e De Azevedo (2009), as camadas normalmente compactadas são esperadas em profundidades mais rasas, o que não é o caso do intervalo avaliado do poço $\mathrm{Y}$, que se encontra a profundidades superiores a $1.000 \mathrm{~m}$. Portanto, a curva de compactação teve comportamento semelhante ao poço Y para o intervalo anormalmente compactado.

Quanto aos métodos da razão e da profundidade equivalente, as análises são similares ao poço X. Nas Figuras 7 e 8 , é possível constatar que o método da razão se apresentou constante para todas as profundidades, o que é incoerente pelos motivos já discutidos na análise do poço $\mathrm{X}$, enquanto o método da PE apresentou resultados totalmente discrepantes dos dados medidos nos testes de RFT e dos resultados preditos pelos demais métodos indiretos. Portanto, pela análise qualitativa das curvas, esses métodos não seriam adequados para previsão do gradiente de poros na área em estudo.

Para a análise quantitativa foram determinados os EA e ER para o poço Y. A análise de erro denunciou que, para os resultados obtidos, os métodos indiretos geraram erros relativamente altos entre eles, inviabilizando-os para esse poço. A Tabela 5 mostra um resumo dos valores de ER médio de cada método, possibilitando um comparativo entre eles.

Da Tabela 5, é possível concluir que, quantitativamente, nenhum método foi capaz de predizer, de forma satisfatória,

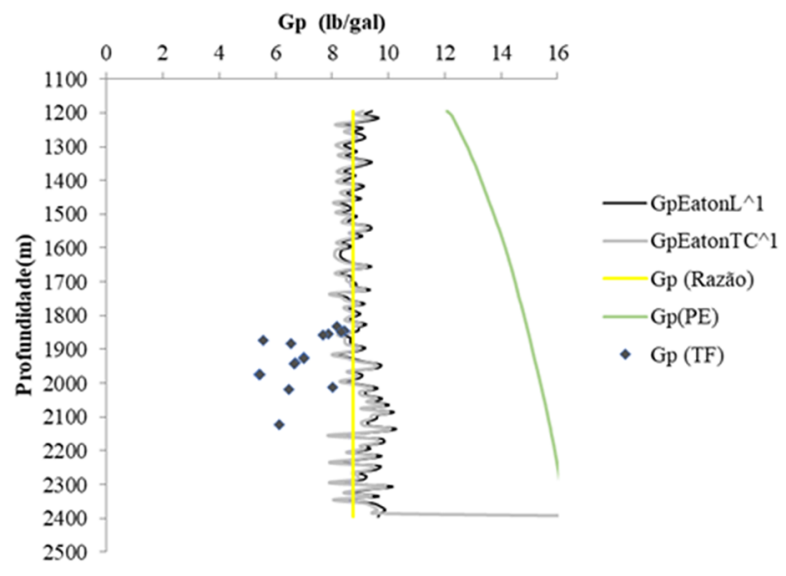

GP: gradiente de poros; L: linear; TC: trend curvo; PE: profundidade equivalente; TF: teste de formação.

Figura 8. Janela operacional dos métodos indiretos do poço $\mathrm{Y}$ utilizando o expoente 1 para o método de Eaton. 
os gradientes medidos. No entanto, se consideradas apenas as profundidades onde o teste de formação mediu os gradientes de poros normais $(1831,1 ; 1844,7$ e 1847,4 m), os ER para o método de Eaton são satisfatórios, independentemente do trend utilizado, seja curvo ou linear, pois apresentaram valores abaixo de $10 \%$.

Como os métodos de previsão de gradiente de poros baseiam-se no mecanismo de subcompactação, eles não foram capazes de prever os gradientes de poros das demais profundidades que têm características de ambiente de pressão de poros anormalmente baixa. Dessa forma, considerando-se apenas os trechos nos quais o gradiente de poros é normal, o método de Eaton foi capaz de prever o gradiente de forma satisfatória. Uma das possíveis razões para ter ocorrido o registro anormal das pressões de poros ao longo da profundidade é a depleção do reservatório devido à presença de outros poços drenando o fluido do mesmo reservatório ou à presença de zonas fraturadas, bem como falhas não seladas, que fazem com que os fluidos não fiquem devidamente confinados dentro dos poros, gerando zonas com pressões de poros anormalmente baixas.

De acordo com Lima Neto (1999), o regime tectônico da Bacia Sergipe-Alagoas apresenta um predomínio de regime compressivo, apresentando falhas reversas. Isso pode implicar na migração dos fluidos para camadas mais rasas e, consequentemente, pode ocorrer a criação de zonas com pressão de poros anormalmente baixa. No entanto, como a Bacia Sergipe-Alagoas já está em produção há muito tempo, a justificativa mais plausível é a existência de outros poços na área, que tenham drenado os fluidos e, como os métodos indiretos não levam em consideração esses fatores, não conseguiram fazer uma boa previsão para a zona na qual o gradiente de poros encontra-se anormalmente baixo.

\section{CONCLUSÕES}

Neste trabalho foram avaliados métodos indiretos de predição do Gp e os valores calculados foram confrontados

Tabela 5. Análise total de erro do poço Y.

\begin{tabular}{lcc}
\hline Método indireto & $\begin{array}{c}\text { Erro absoluto } \\
\text { (médio) }\end{array}$ & $\begin{array}{c}\text { Erro relativo \% } \\
\text { (médio) }\end{array}$ \\
\hline EatonTC (expoente 3) & 1,85 & 29,37 \\
EatonTC (expoente 1) & 1,82 & 28,86 \\
EatonL (expoente 3) & 2,24 & 35,25 \\
EatonL (expoente 1) & 1,93 & 30,64 \\
Razão & 1,78 & 28,29 \\
PE & 5,44 & 82,31 \\
\hline
\end{tabular}

ETC: trend curvo; L: linear; PE: profundidade equivalente. com dados diretos medidos nos poços. Para os poços $\mathrm{X}$ e Y, o método de Eaton (linear ou trend curvo) foi o mais apropriado para a previsão do Gp na Bacia Sergipe-Alagoas. Dentre as metodologias apresentadas para predição do gradiente de poros pelo método de Eaton, a de trend linear se mostrou mais adequada para emprego na área em estudo, pois apresenta os menores ER. Em ambos os poços, o método da profundidade equivalente não foi adequado para o cálculo indireto do Gp, pois obteve o maior ER, assim como o método da razão, que, apesar de fornecer erros ER baixos, apresentou comportamento incoerente com o mecanismo de subcompactação. Para o poço X, e utilizando-se o método de Eaton com expoente 3, a escolha da metodologia trend linear aproximou os valores preditos dos dados medidos nos testes de RFT, diminuindo seu ER. Os resultados mostraram ser necessária uma análise qualitativa e quantitativa para definição do melhor método indireto de previsão do gradiente de poros. Além disso, os resultados obtidos concordaram com estudos já efetuados, indicando ser o expoente de Eaton igual a 1 o mais adequado para a área em estudo.

\section{REFERÊNCIAS}

Bowers, G. (1995). Pore pressure estimation from velocity data. Accounting for Overpressure Mechanisms Besides Undercompaction. SPE Drilling \& Completion, 10, 89-95.

De Andrade, J. F. (2014). Avaliação da aplicabilidade de correlações empíricas desenvolvidas para o Golfo do México em projetos de poços da bacia Sergipe-Alagoas. Trabalho de Conclusão de Curso (Graduação). São Cristóvão: Universidade Federal de Sergipe.

Doyen, L., Gaudoin, O. (2004). Classes of imperfect repair models based on reduction of failure intensity or virtual age. Reliability Engineering and System Safety, 84(1), 45-56.

Dutta, N. C. (2002). Geopressure prediction using seismic data: Current status and the road ahead. Geophysics, 67(6), 2012-2041.

Eaton, B. A. (1975). The equation for geopressure prediction from well logs. Society of Petroleum Engineers. 11 p.

Ferreira, C. D. (2010). Novo método para estimativa do gradiente de fratura para poços de petróleo. Tese (Doutorado). Natal: Universidade Federal do Rio Grande do Norte.

Foster, J. B. (1966). Estimation of formation pressures from electrical surveys-Offshore Louisiana. Journal of Petroleum Technology, 2, 165-171. 
Holbrook, P. W., Maggiori, D. A., Hensley, R. (1995). Realtime pore pressure and fracture pressure determination in all sedimentar lithologies. Society of Petroleum Engineers Formation Evaluation, 10(4), 215-222.

Hottman, C. E., Johnson, R. K. (1965) Estimation of formation pressures from log-derived shale properties. Journal of Petroleum Technology, 17, 717-722.

Issler, D. R. (1992). A new approach to shale compaction and stratigraphic restoration, Beaufort-Mackenzie Basin and Mackenzie Corridor, northern Canada. American Association of Petroleum Geologists Bulletin, 76, 1170-1189.

Lima Neto, F. F. (1999). Regime atual de tensões nas bacias sedimentares brasileiras. VII Simpósio Nacional de Estudos Tectônicos. Lençóis: SBG.
Pereira, B. C. M. (2007). Proposta de uma metodologia para estimativa de geopressões. Dissertação (Mestrado). Rio de Janeiro: Universidade Federal do Rio de Janeiro.

Pennebaker, E. S. (1968). An engineering interpretation of seismic data. Society of Petroleum Engineers.

Rocha, L. A. S., De Azevedo, C. T. (2009). Projetos de poços de petróleo: geopressões e assentamento de colunas de revestimentos. $2^{\mathrm{a}}$ ed. Rio de Janeiro: Interciência.

Santos, J.P. L., Andrade, J. F., Oliveira, R. C., Almeida Neto, J. B., Santos,A.P.P.(2015). Projetos de poços: um estudo de casona bacia Sergipe-Alagoas. Revista Eletrônica de Petróleo e Gás, 3, 31-40.

Sayers, C. M., Johnson, G. M., Denyer, G. (2002). Predrill pore pressure prediction using seismic data. Geophysics, 67, 1286-1292. 